\title{
Comparison of Magnetic Resonant Coupling Wireless Power Transfer Systems within Aligned and Unaligned Positions and Determining their Limits
}

\author{
Ali Agcal, Nur Bekiroglu, and Selin Ozcira* \\ Electrical Engineering Department, Yildiz Technical University, Davutpasa, 34220, Istanbul, Turkey
}

(Received 15 July 2016, Received in final form 1 November 2016, Accepted 22 November 2016)

\begin{abstract}
In this study, the efficiencies for both the angular aligned and unaligned positions of the receiver and transmitter coils of wireless power transfer (WPT) systems are examined. Some parameters of the equivalent circuit were calculated with Maxwell 3D software. The analytical solution of the circuit was calculated in MATLAB program through the composition of the system's mathematical modeling. The numerical solution of the system, however, was calculated using PSIM, which is circuit simulation software. In addition, with the use of the finite element method (FEM) in Maxwell 3D software, transient analysis of the three-dimensional system was performed. The efficiency of the system was estimated through the calculation of input and output power. The results demonstrated that power was efficiently transmitted to a certain extent in aligned and unaligned positions. The results also revealed that, for aligned positions, high efficiency with air gaps of $15-20 \mathrm{~cm}$ can be obtained and that the efficiency quickly dropped with air gaps of more than $20 \mathrm{~cm}$. For spatially unaligned positions, it was observed that wireless power transfer could be realized with high efficiency with air gaps of up to $10 \mathrm{~cm}$ and that efficiency quickly dropped with air gaps of more than $10 \mathrm{~cm}$.
\end{abstract}

Keywords : wireless power transfer, magnetic resonant coupling, alignment of coils, system efficiency

\section{Introduction}

Although wireless power transfer (WPT) has been a research topic for more than 200 years, it has not been actualized in the culture of electrical and electronic engineering, which can be seen as a critical problem. Since it was first proposed by Nicola Tesla in 1890, WPT has gradually increased in importance with the progress of technology as a multidisciplinary field and as a shared domain for different branches of science [1,2]. Moreover, WPT plays a substantial role in the implementation of various applications such as electrical appliances, home electronics, and medical devices, all of which can provide solutions for possible problems in the future.

So far, wireless energy transfer has been practiced with electromagnetic radiation, microwaves, lasers, electromagnetic induction, and magnetic resonant coupling. The first studies on WPT were initiated by Nicola Tesla in an attempt to convey wireless electrical energy to the whole world with electromagnetic radiation. However, the studies

(C)The Korean Magnetics Society. All rights reserved.

*Corresponding author: Tel: +90-212-383-5846

Fax:+90-212-383-5858, e-mail: sozcira@yildiz.edu.tr could not be finalized due to the sponsors' withdrawal from the project [1-3].

The idea of using microwaves in WPT emerged with the development of high-powered microwave transmitters after World War II. One of the pioneers in microwave power transfer, William Brown, designed a model helicopter, of which all the cruising power was provided by microwave beams [4].

Sahai and Graham actualized power transfer using lasers, which works at low efficiency in high distances as a type of wireless energy. However, the laser radiation, which is more suitable for space applications, is quite harmful, causing blindness even at low power, and killing living creatures through partial heating at high power [5].

Zhao and his colleagues have conducted research on electromagnetic induction theory, reaching high efficiencies, in which the active working distance from the primary coil to the secondary coil is only a few centimeters. However, the excessive proximity and the difficulty in aligning the secondary coil with the primary coil are the disadvantages of this method [6]. The theory of magnetic resonant coupling started with Massachusetts Institute of Technology (MIT) scientists' invention in 2007, called 'WiTricity', which revolutionized the technology 
of WPT. Using magnetic resonant coupling, a 60 Watt bulb could be lit from a distance of more than 2 meters and without using any wires, achieving roughly $40 \%$ efficiency. In this way, mid-range WPT was actualized. In the WiTricity system, energy is transferred via magnetic resonant coupling. The power in the primary resonance coil is fully utilized by the secondary resonance coil in the optimal operation. Consequently, the power that is transferred can be efficiently shared among resonance objects, and weakly shared among non-resonance objects $[7,8]$.

Since previous researches that utilize inductive power transfer based on magnetic induction theory have low efficiency, their popularity has been dwindled and replaced in recent years by magnetic resonant coupling.

This study demonstrates the differences in the efficiencies of positional and angular aligned and unaligned conditions with respect to different air gaps and different angles. The study also demonstrates the limits of air gaps and angles in the aligned and unaligned positions, the change of mutual inductance and resonance frequency, and the differences in input and output current. The equivalent circuit simulation of the magnetic resonant coupling system was performed using PSIM software. In addition, the receiving and transmitting inductances were designed with Maxwell 3D software. The equivalent circuit of the magnetic resonance coupling system was set up in the Maxwell Circuit Editor (MCE) program. Using the receiver and transmitter coils in the Maxwell 3D software and the circuit in the MCE program, the transient analysis of the WPT system was performed in this study with the finite element method (FEM).

\section{Calculation of Efficiency and Equivalent Impedance with Equivalent Circuit of Magnetic Resonant Coupling}

In this section, the simple theory of the equivalent circuit will be explained. In order to analytically represent

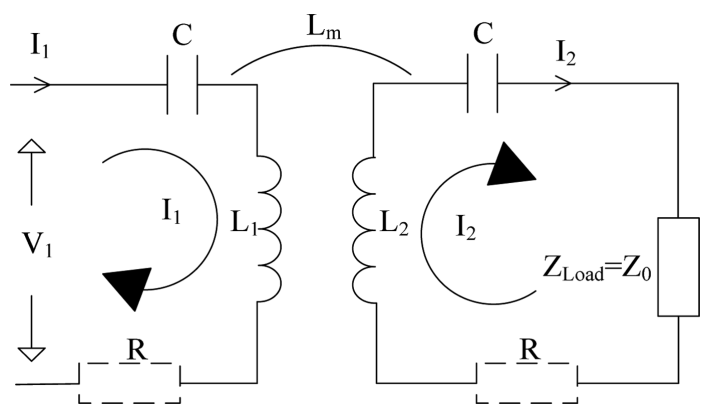

Fig. 1. The equivalent circuit of the wireless power transfer system $[9,10]$. the input impedance, power transfer, and efficiency, a simplified equivalent circuit of the WPT is used, as illustrated in Fig. 1.

In this equivalent circuit, $I_{1}$ represents the input current (transmitting current), $I_{2}$ represents the output current (receiving current), $V_{1}$ represents the input voltage, $R$ represents the internal resistances of the receiving and transmitting system, $C$ represents the receiving and transmitting resonance capacity, $L_{1}$ represents the transmitter coil, $L_{2}$ represents the receiver coil, $L_{m}$ represents the mutual inductance, and $Z_{\text {load }}\left(Z_{0}\right)$ represents the load impedance (characteristic impedance). To simplify the calculations of the resonant frequencies, $R$ is ignored. The circuit shown in Fig. 1 is a model of a wireless power system created using a pair of magnetically coupled resonators. To calculate the resonant frequencies, it is advantageous to derive an alternative equivalent circuit.

Eq. (1) illustrates the circumference of the transmitting part, while Eq. (2) depicts the circumference equation of the receiving part.

$$
\begin{aligned}
& V_{1}=I_{1}\left(R+j L_{1} \omega+\left(\frac{1}{j \omega C}\right)\right)-I_{2}\left(j L_{m} \omega\right) \\
& 0=I_{2}\left(j L_{2} \omega+\left(\frac{1}{j \omega C}\right)+Z_{0}+R\right)-I_{1}\left(j L_{m} \omega\right)
\end{aligned}
$$

From Eq. (2), the relationship between the currents of receiver and transmitter coils can be derived as shown in Eq. (3).

$$
I_{2}=I_{1} \frac{j L_{m} \omega}{j L_{2} \omega+\left(\frac{1}{j \omega C}\right)+Z_{0}+R}
$$

The equivalent impedance can be obtained by placing Eq. (3) in Eq. (1) and dividing the generated voltage formula by the input current as to obtain Eq. (4).

$$
Z_{e q}=Z_{i n}=R+j L_{1} \omega+\left(\frac{1}{j \omega C}\right)+\frac{L_{m}{ }^{2} \omega^{2}}{j L_{2} \omega+\left(\frac{1}{j \omega C}\right)+Z_{0}+R}
$$

The equivalent circuit in Fig. 1 can also be shown with

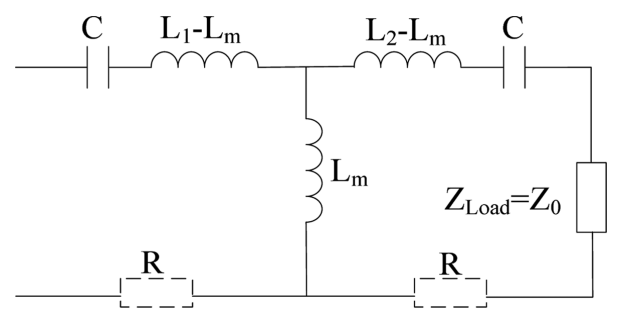

Fig. 2. T-type equivalent circuit of the WPT system. 
a T-type equivalent circuit, as shown in Fig. 2. It is assumed that the circuit represented in Fig. 1 is equivalent to the circuit represented in Fig. 2. This assumption leads to the satisfaction of the symmetric condition given by $Z_{0}=Z_{\text {load }}$.

The efficiency of this system is shown in Eq. (5).

$$
\eta=\frac{P_{\text {out }}}{P_{\text {in }}}=\frac{I_{\text {out }}^{2} Z_{\text {out }}}{I_{\text {in }}^{2} Z_{\text {in }}}
$$

Here, the output current $\left(I_{\text {output }}\right)$ is equal to the current that flows through the receiver $\left(I_{2}\right)$, the input current $\left(I_{\text {input }}\right)$ is equivalent to the current that flows through the transmitter $\left(I_{1}\right)$, and $Z_{\text {output }}$ impedance equals to $Z_{\text {load }}$ impedance. If we show Eq. (3) as the proportion of the output current to the input current, we can derive Eq. (6).

$$
\frac{I_{\text {out }}}{I_{\text {in }}}=\frac{j L_{m} \omega}{j L_{2} \omega+\left(\frac{1}{j \omega C}\right)+Z_{0}+R}
$$

When Eq. (6) and Eq. (4) are inserted in Eq. (5), the efficiency formula can be derived as in Eq. (7).

$$
\begin{aligned}
\eta=\left\{\frac{j L_{m} \omega}{j L_{2} \omega+\left(\frac{1}{j \omega C}\right)+Z_{0}+R}\right\}^{2} & \frac{Z_{0}}{R+j L_{1} \omega+\left(\frac{1}{j \omega C}\right)+\left\{\frac{L_{m}{ }^{2} \omega^{2}}{j L_{2} \omega+\left(\frac{1}{j \omega C}\right)+Z_{0}+R}\right\}}
\end{aligned}
$$

$L_{m}^{2}=\frac{Z_{0}^{2}-R^{2}}{\omega_{0}^{2}}$

$$
\begin{aligned}
& L_{m}^{2}>\frac{Z_{0}^{2}-R^{2}}{\omega_{0}^{2}} \\
& L_{m}^{2}<\frac{Z_{0}^{2}-R^{2}}{\omega_{0}^{2}}
\end{aligned}
$$

$\omega_{0}$ is the resonance frequency, $L_{m}$ denotes the mutual inductance for maximum efficiency, $L$ denotes the receiving and transmitting inductance, $Z_{0}$ denotes the characteristic impedance, and $R$ refers to the internal resistance conditions in Eq. (7). $L_{m}$ is related to the air gap, and is therefore calculated for different air gap values and it needs to be calculated severally for the individual characteristic impedance values. Resonance frequencies are detected by solving Eq. (9). In Eq. (9), the system possesses double-resonance frequency. When the system is over-coupled to achieve maximum efficiency at double resonance frequency condition, the lower value of $\omega_{0}$ is used in order to achieve lower $Z_{e q}$. In Eq. (10), however, the system has single-resonance frequency with low efficiency [11]. Actually, Eq. (9) shows that single-resonance frequency occurs in places where the air gap is large and double-resonance frequency occurs where the air gap is narrow. Eq. (8), on the other hand, demonstrates the limits in the emergence of single or double-resonance frequency in this system.

\section{Parameter Calculation using Analytics and Finite Elements Method by Setting Equivalent Circuit of The WPT System}

This section discusses the use of the MATLAB program for the analytical solution of the circuit [12], the use of PSIM for the solution of the equivalent circuit [13], and the use of Maxwell 3D software for FEM [14]. The parameters of the circuit were calculated by Maxwell 3D software. In this study, the coils are designed in Maxwell

Table 1. System parameters obtained by Maxwell 3D Software in aligned position.

\begin{tabular}{cccccccccc}
\hline \hline Parameter/Air Gap & $1 \mathrm{~cm}$ & $5 \mathrm{~cm}$ & $10 \mathrm{~cm}$ & $15 \mathrm{~cm}$ & $20 \mathrm{~cm}$ & $30 \mathrm{~cm}$ & $40 \mathrm{~cm}$ & $50 \mathrm{~cm}$ \\
\hline$L[\mathrm{nH}]$ & 981.9 & 982.4 & 983.8 & 1010.5 & 991 & 994 & 1014.8 & 1011.1 \\
$L_{m}[\mathrm{nH}]$ & 548.5 & 241.8 & 128.3 & 80.5 & 46.4 & 20.9 & 11.1 & 5.9 & 124 \\
$C[\mathrm{pF}]$ & 124 & 124 & 124 & 124 & 124 & 124 & 124 \\
\hline
\end{tabular}

Table 2. System parameters obtained by Maxwell 3D Software in unaligned position.

\begin{tabular}{ccccccccc}
\hline \hline Parameter/Air Gap & $1 \mathrm{~cm}$ & $5 \mathrm{~cm}$ & $10 \mathrm{~cm}$ & $15 \mathrm{~cm}$ & $20 \mathrm{~cm}$ & $30 \mathrm{~cm}$ & $40 \mathrm{~cm}$ & $50 \mathrm{~cm}$ \\
\hline$L[\mathrm{nH}]$ & 1040 & 1000.8 & 996.5 & 988.8 & 1020.1 & 996.6 & 993.5 & 1009.1 \\
$L_{m}[\mathrm{nH}]$ & 118 & 81.6 & 56.3 & 38.3 & 26.3 & 14 & 8.2 & 4.8 \\
$C[\mathrm{pF}]$ & 124 & 124 & 124 & 124 & 124 & 124 & 124 & 124 \\
\hline
\end{tabular}




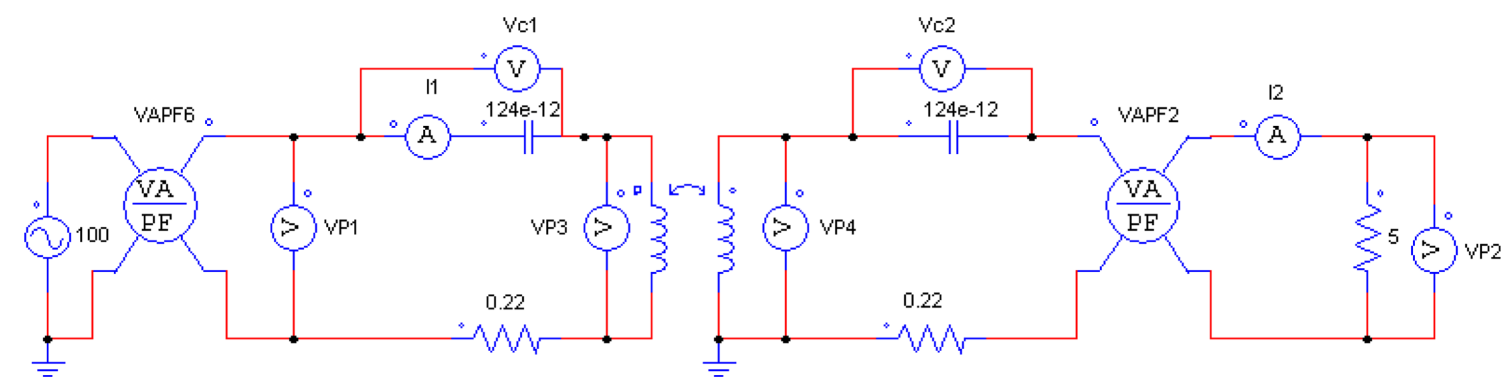

Fig. 3. (Color online) Equivalent circuit of magnetic resonant coupling in PSIM Circuit Simulation Software.
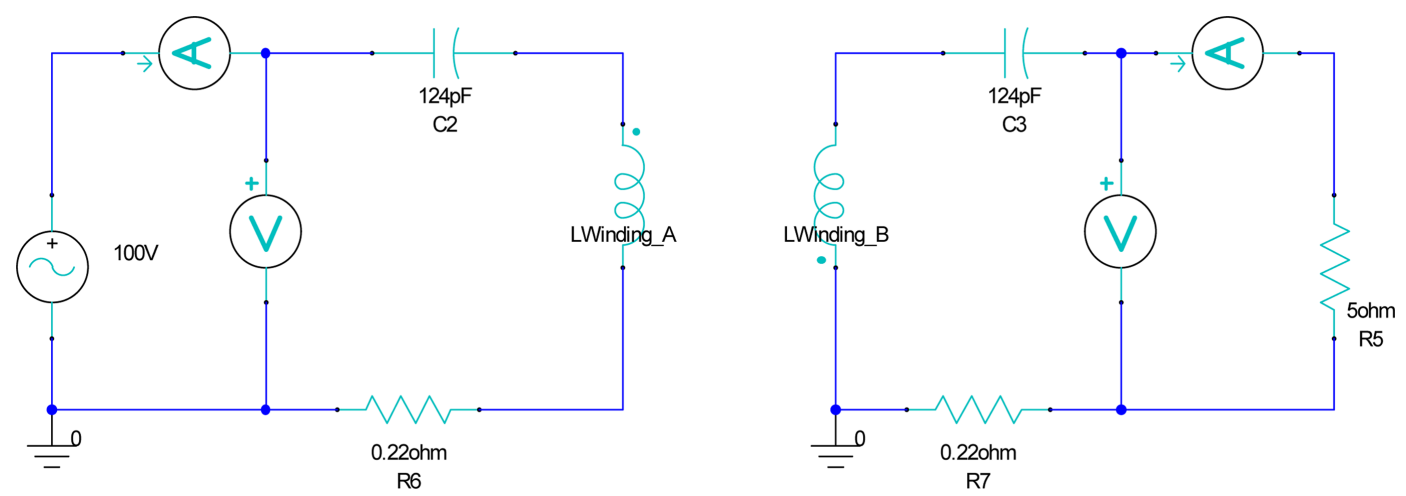

Fig. 4. (Color online) Maxwell Circuit Editor Model.

3D. The inductances of coils and the mutual inductance between two coils are calculated by Maxwell 3D. After calculating inductance, the resonance frequencies are calculated by the MATLAB program and the circuit of the WPT system is simulated with PSIM and Maxwell 3D.

\subsection{Comparing Spatially Aligned and Unaligned Posi-} tions

In the system, $L$ is a nonlinear parameter and varies according to the current. Each parameter of the system for the aligned position was obtained as shown in Table 1 .

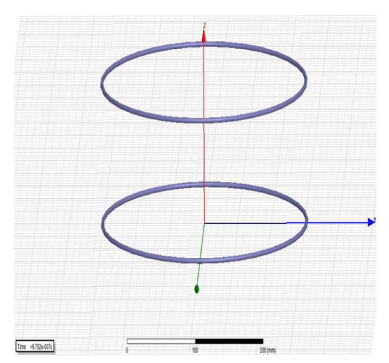

(a)

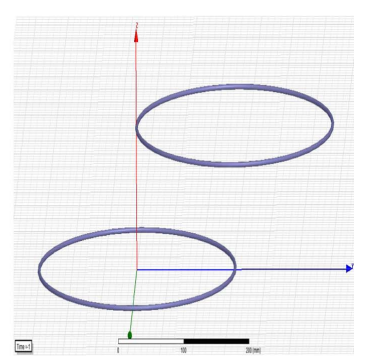

(b)
Fig. 5. (Color online) Aligned (a) and unaligned (b) Maxwell 3D Models of receiver and transmitter.

Table 3. Efficiency of WPT system established in aligned and unaligned positions for different air gaps.

\begin{tabular}{lcccccccc}
\hline \hline Alinged & \multicolumn{7}{c}{ Efficiency (\%) } \\
\hline Air Gap & $1 \mathrm{~cm}$ & $5 \mathrm{~cm}$ & $10 \mathrm{~cm}$ & $15 \mathrm{~cm}$ & $20 \mathrm{~cm}$ & $30 \mathrm{~cm}$ & $40 \mathrm{~cm}$ & $50 \mathrm{~cm}$ \\
\hline MATLAB & 91.75 & 91.75 & 91.75 & 91.75 & 87.15 & 37.29 & 12.21 & 3.63 \\
PSIM & 90.94 & 91.93 & 91.93 & 91.80 & 89.80 & 72.01 & 43.22 & 17.77 \\
Maxwell 3D & 66.89 & 67.79 & 65.29 & 71.30 & 62.76 & 21.07 & 7.38 & 1.42 \\
\hline Unalinged & & & \multicolumn{7}{c}{ Efficiency (\%) } & \\
\hline Air Gap & $1 \mathrm{~cm}$ & $5 \mathrm{~cm}$ & $10 \mathrm{~cm}$ & $15 \mathrm{~cm}$ & $20 \mathrm{~cm}$ & $30 \mathrm{~cm}$ & $40 \mathrm{~cm}$ & $50 \mathrm{~cm}$ \\
MATLAB & 91.75 & 91.75 & 91.66 & 77.88 & 51.21 & 19.13 & 7.00 & 2.45 \\
PSIM & 91.90 & 91.90 & 91.71 & 87.3 & 79.23 & 55.07 & 29.91 & 12.71 \\
Maxwell 3D & 65.49 & 69.67 & 66.05 & 53.45 & 33.44 & 11.87 & 4.19 & 1.42 \\
\hline
\end{tabular}


For the unaligned position, each parameter of the system was obtained as shown in Table 2.

In the circuit simulation, the WPT system was fed from a sinusoidal voltage source, the peak voltage of which was $100 \mathrm{~V}$. The model illustrated in Fig. 3 was completed in PSIM circuit simulation software.

The same circuit was modeled as shown in Fig. 4 with the Maxwell Circuit Editor (MCE) program. The circuits of the receiver and transmitter coils were modeled with

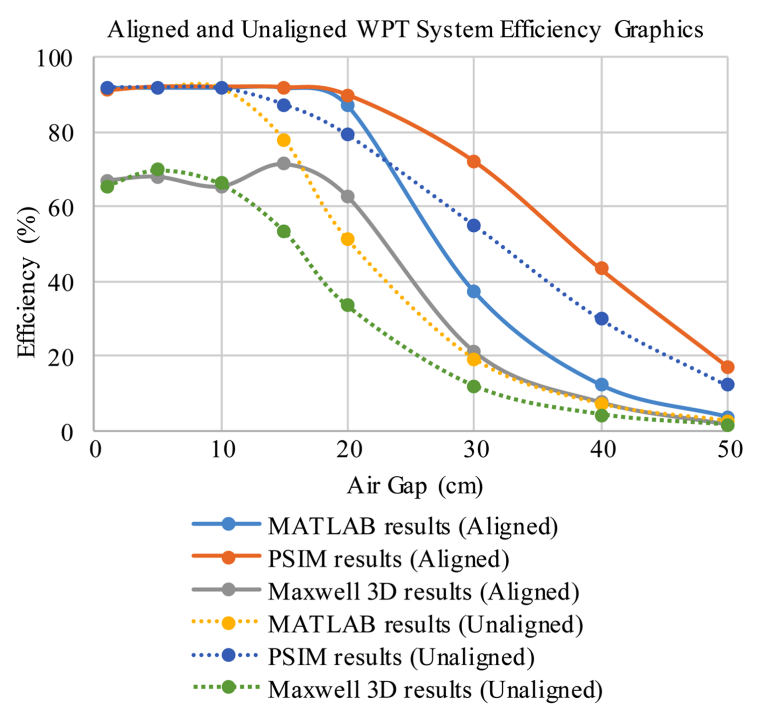

Fig. 6. (Color online) Graphs of efficiency for aligned and unaligned positions varying by different air gaps. the MCE program, while the receiver and transmitter coils were modeled with the Maxwell 3D program, as shown in Fig. 5(a) and Fig. 5(b).

The efficiency of the WPT system that works with magnetic resonant coupling, established in aligned and unaligned positions for different air gaps, is shown in 3 separate programs in Table 3. In addition, the change of the efficiency with respect to the air gap is illustrated in Fig. 6.

The distributions of the magnetic flux are modeled with Maxwell 3D, as shown in Fig. 7. For each air gap value and positional aligned and unaligned condition, the input and output flow of the system, the peak voltage to which the capacities of receiving and transmitting resonance are exposed, the input and output voltage, and the input and output power are shown in Table 4.

\subsection{Comparison of Aligned and Unaligned Positions}

This section discusses the efficiency rates for angularly aligned positions and for positions which are not aligned according to different angles. Here, an air gap of $10 \mathrm{~cm}$ was chosen. This is because the receiver and the transmitter intertwine when the angles of alignment in the system are changed in air gaps narrower than $10 \mathrm{~cm}$. In the system, $\mathrm{L}$ is a parameter that is not linear and varies according to the current of the system. Each parameter of the system for angularly aligned and unaligned positions

Table 4. Input and output current, voltage and power of the WPT system obtained in aligned and unaligned positions for different air gaps.

\begin{tabular}{|c|c|c|c|c|c|c|c|c|}
\hline \multirow{2}{*}{$\begin{array}{l}\text { Maxwell 3D } \\
\text { Air Gap }\end{array}$} & \multicolumn{8}{|c|}{ Aligned Results } \\
\hline & $1 \mathrm{~cm}$ & $5 \mathrm{~cm}$ & $10 \mathrm{~cm}$ & $15 \mathrm{~cm}$ & $20 \mathrm{~cm}$ & $30 \mathrm{~cm}$ & $40 \mathrm{~cm}$ & $50 \mathrm{~cm}$ \\
\hline Transmitter Current (A) & 8.89 & 10.15 & 10.69 & 8.90 & 18.05 & 40.19 & 45.99 & 48.34 \\
\hline Receiver Current (A) & 8.48 & 9.87 & 9.75 & 9.43 & 12.63 & 10.97 & 6.87 & 3.45 \\
\hline Input Voltage (V) & 70.71 & 70.71 & 70.71 & 70.71 & 70.71 & 70.71 & 70.71 & 70.71 \\
\hline Output Voltage (V) & 42.42 & 49.35 & 48.75 & 47.14 & 63.14 & 54.83 & 34.35 & 17.24 \\
\hline Input Power (W) & 1158 & 1430.5 & 1461 & 1246.9 & 2549 & 5707 & 6503 & 6835 \\
\hline Output Power (W) & 720 & 974 & 953.6 & 889 & 1595 & 1203 & 480 & 121 \\
\hline Transmitter Capacitor Peak (V) & 1357 & 1411 & 1397 & 1150 & 2283 & 5071 & 5867 & 6153 \\
\hline Receiver Capacitor Peak (V) & 1346 & 1383 & 1307 & 1239 & 1604 & 1387 & 892 & 447 \\
\hline Maxwell 3D & \multicolumn{8}{|c|}{ Unaligned Results } \\
\hline Air Gap & $1 \mathrm{~cm}$ & $5 \mathrm{~cm}$ & $10 \mathrm{~cm}$ & $15 \mathrm{~cm}$ & $20 \mathrm{~cm}$ & $30 \mathrm{~cm}$ & $40 \mathrm{~cm}$ & $50 \mathrm{~cm}$ \\
\hline Transmitter Current (A) & 12.08 & 10.56 & 13.81 & 24.26 & 34.54 & 44.34 & 47.59 & 48.53 \\
\hline Receiver Current (A) & 10.52 & 10.17 & $11.3 \mathrm{~A}$ & 13.52 & 12.72 & 8.65 & 5.33 & 3.12 \\
\hline Input Voltage (V) & 70.71 & 70.71 & 70.71 & 70.71 & 70.71 & 70.71 & 70.71 & 70.71 \\
\hline Output Voltage (V) & 52.59 & 50.86 & 56.51 & 67.62 & 63.62 & 43.26 & 26.65 & 15.62 \\
\hline Input Power (V) & 1689 & 1485 & 1934 & 3422 & 4885 & 6304 & 6772 & 6861 \\
\hline Output Power (W) & 1106 & 1035 & 1277 & 1829 & 1634 & 748 & 284 & 97 \\
\hline Transmitter Capacitor Peak (V) & 1619 & 1368 & 1733 & 3053 & 4423 & 5606 & 6006 & 6172 \\
\hline Receiver Capacitor Peak (V) & 1428 & 1330 & 1435 & 1709 & 1646 & 1095 & 673 & 397 \\
\hline
\end{tabular}




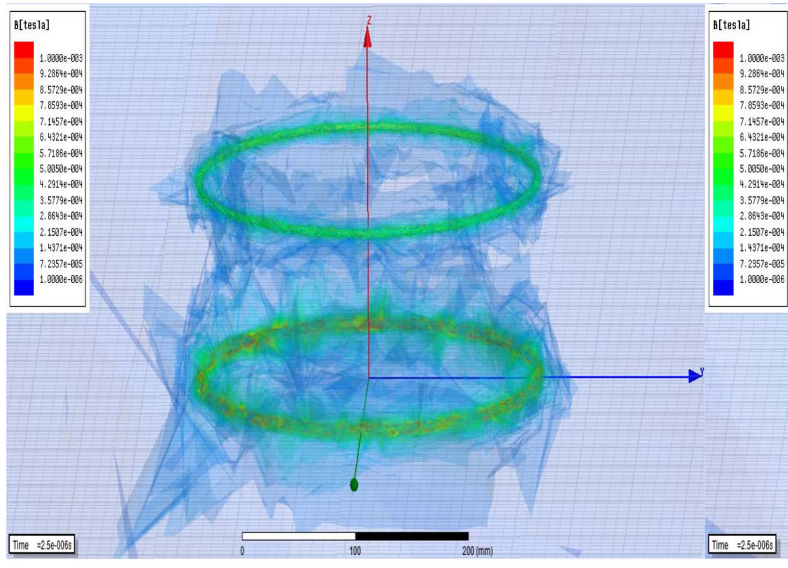

(a)

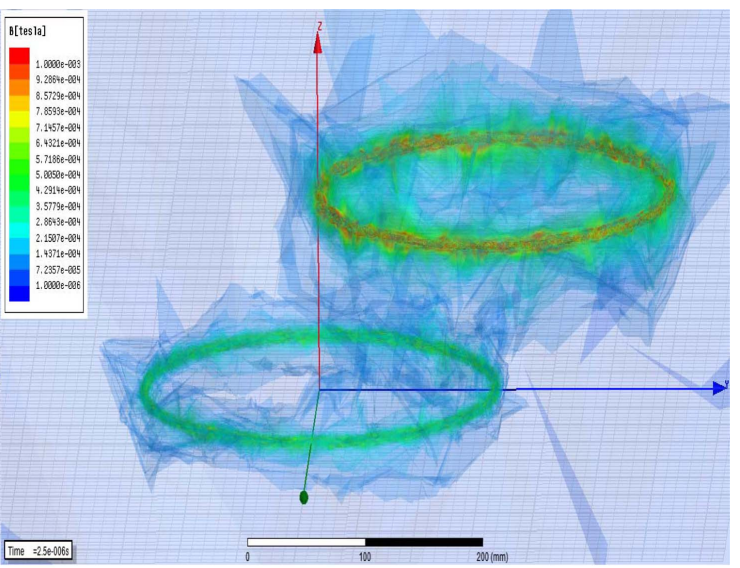

(b)

Fig. 7. (Color online) Distributions of the magnetic flux density both aligned and unaligned positions.

Table 5. System parameters obtained by Maxwell 3D software in the different angle positions.

\begin{tabular}{ccccc}
\hline \hline Parameter/Angle & $0^{\circ}$ & $10^{\circ}$ & $20^{\circ}$ & $30^{\circ}$ \\
\hline$L[\mathrm{nH}]$ & 983.8 & 1023.2 & 1000.3 & 984.5 \\
$L_{m}[\mathrm{nH}]$ & 128.3 & 6.8 & 2.7 & 1.5 \\
$C[\mathrm{pF}]$ & 124 & 124 & 124 & 124 \\
\hline
\end{tabular}

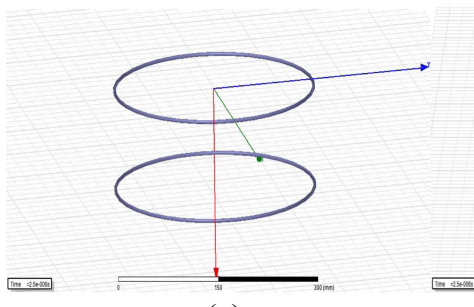

(a)

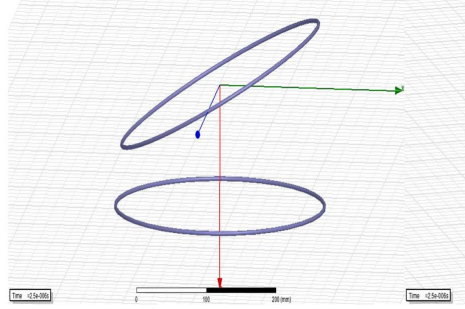

(c)

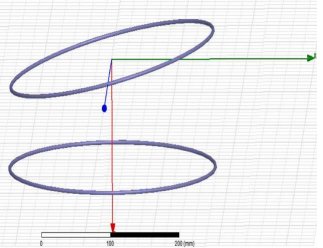

(b)

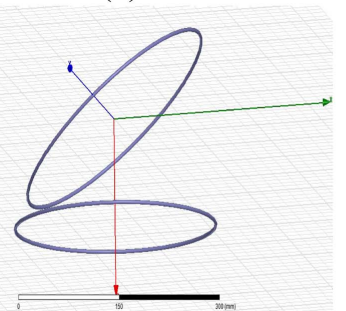

(d)
Fig. 8. (Color online) Receiving and transmitting positions of Maxwell 3D: which is Aligned (a), $10^{\circ}$ Unaligned (b), $20^{\circ}$ Unaligned (b) and $30^{\circ}$ Unaligned (d).

was calculated as shown in Table 5.

In the circuit simulation, the WPT system was directly fed with a sinusoidal voltage source, the peak voltage was $100 \mathrm{~V}$, as shown in Fig. 3. The model study illustrated in Fig. 3 was completed by PSIM circuit simulation software.

The same circuit was modeled as shown in Fig. 4 in the
Table 6. Efficiency rates of the WPT system for angularly aligned and unaligned positons.

\begin{tabular}{|c|c|c|c|c|}
\hline $10 \mathrm{~cm}$ Air Gap & & Efficie & cy $(\%)$ & \\
\hline Angle & $0^{\circ}$ & $10^{\circ}$ & $20^{\circ}$ & $30^{\circ}$ \\
\hline $\begin{array}{l}\text { MATLAB } \\
\text { solved equations results }\end{array}$ & 91.75 & 23.07 & 4.66 & 0.756 \\
\hline PSIM results & 91.93 & 23.07 & 4.64 & 1.5153 \\
\hline Maxwell 3D results & 65.29 & 0.0144 & 0.0026 & 0.0007 \\
\hline
\end{tabular}

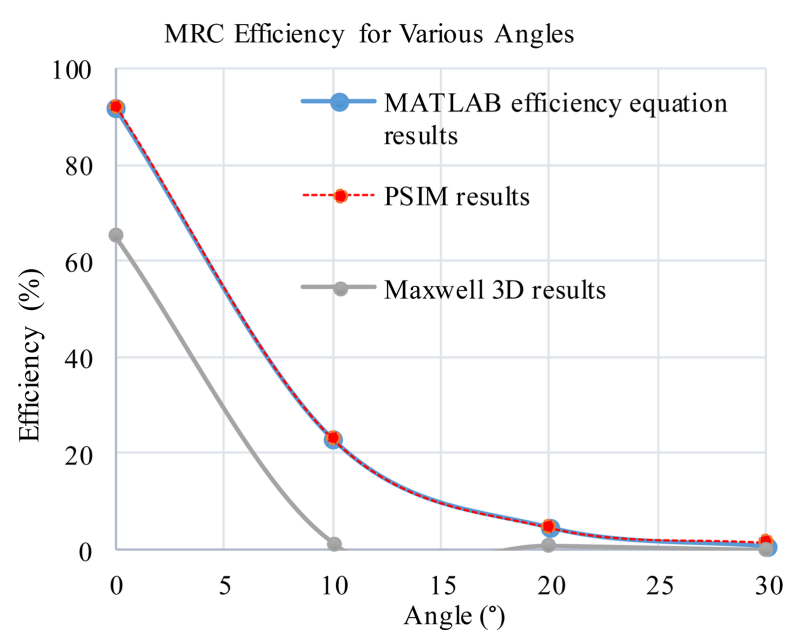

Fig. 9. (Color online) Efficiency for various angles.

MCE program. The receiver and transmitter coils were modeled in the Maxwell 3D program as shown in Fig. 8, and the circuits of the receiver and transmitter coils were modeled in the MCE program.

The efficiency rates of the WPT systems that work with magnetic resonant coupling are shown in 3 different programs in Table 6. The change of efficiency with respect to the angle is also illustrated in Fig. 9. 


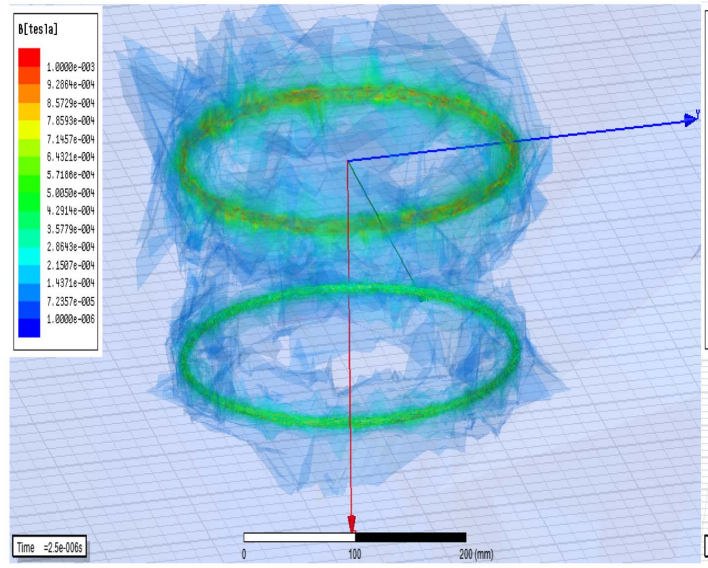

(a)

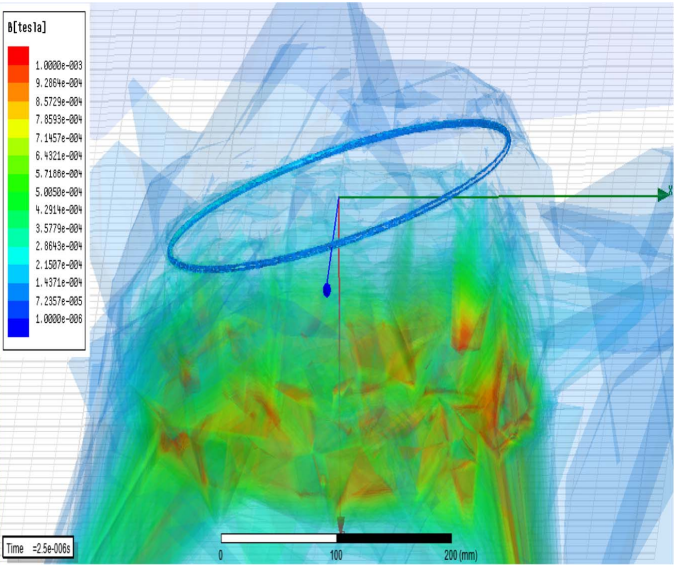

(b)

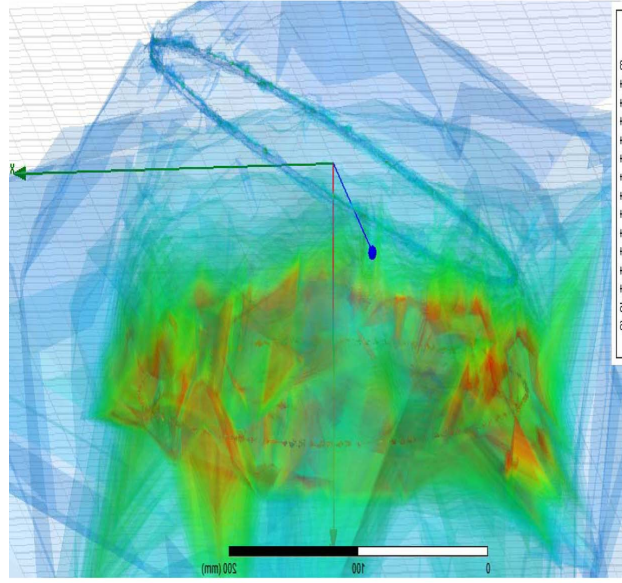

(c)

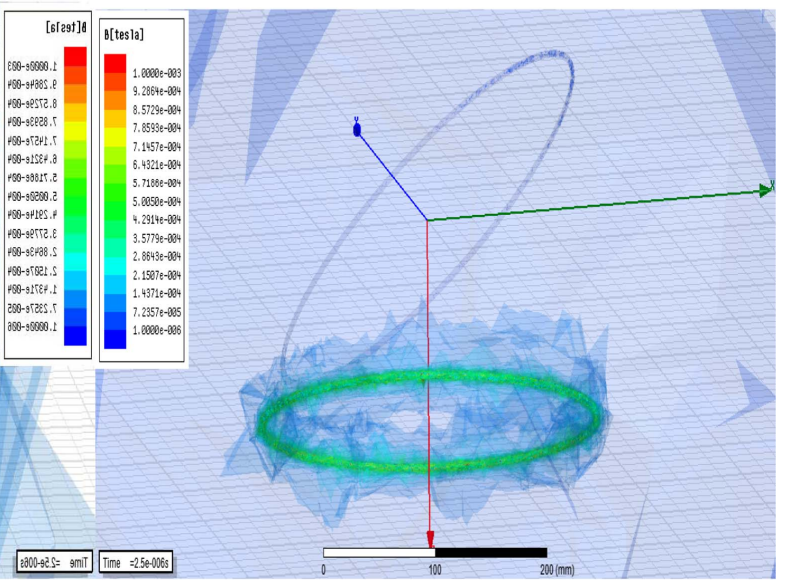

(d)

Fig. 10. (Color online) Distribution of magnetic flux density: Aligned (a), $10^{\circ}$ Unaligned (b), $20^{\circ}$ Unaligned (c) and $30^{\circ}$ Unaligned (d).

Table 7. Input and output current, voltage and power values obtained in aligned and unaligned positions for different angles for $10 \mathrm{~cm}$ air gap in Maxwell 3D.

\begin{tabular}{lcccc}
\hline \hline Angle & $0^{\circ}$ & $10^{\circ}$ & $20^{\circ}$ & $30^{\circ}$ \\
\hline Transmitter Current (A) & 10.69 & 48.96 & 49.24 & 49.44 \\
Receiver Current (A) & 9.75 & 0.33 & 0.19 & 0.1 \\
Input Voltage (V) & 70.71 & 70.71 & 70.71 & 70.71 \\
Output Voltage (V) & 48.75 & 1.63 & 0,935 & 0,525 \\
Input Power (W) & 1461 & 6924 & 6964 & 7040 \\
Output Power (W) & 953.6 & 1 & 0.18 & 0.05 \\
Transmitter Capacitor Peak (V) & 1397 & 6268 & 6233 & 6294 \\
Receiver Capacitor Peak (V) & 1307 & 41.9 & 16.8 & 9.3 \\
\hline
\end{tabular}

Distributions of the magnetic flux with respect to different angles of alignment are modeled with Maxwell 3D, as shown in Fig. 10.

With Maxwell 3D software, the input and output currents of the system, the peak voltage to which the capacities of the receiving and transmitting resonance are exposed, the input and output voltages, and the angularly aligned and unaligned positions are illustrated in Table 7.

Corresponding to some of the definite geometrical dimensions of the WPT system, the efficiency and power values are very close to zero, when the alignment angle increases. This should be avoided in the design of the system. Indeed, according to the software used, the efficiency rates vary. While the difference is almost negligible for MATLAB and PSIM, which enables numerical analysis, the Maxwell 3D software results are considerably disparate owing to the analytic solver ground.

When the alignment angle is small, the distribution of magnetic flux density is roughly uniform and the power ratio is reasonable; as the angle is increased, the efficiency decreases due to the scattered magnetic flux density.

\section{Conclusion}

This study shows the limits of positional and angular 
aligned and unaligned conditions for the WPT system which works with magnetic resonant coupling. In the design, it was observed that, for aligned positions, power could be transferred with high efficiency within air gaps of $15-20 \mathrm{~cm}$ and that efficiency quickly decreased within air gaps of more than $20 \mathrm{~cm}$. For the spatially unaligned positions, it was observed that WPT could be realized with high efficiency with air gaps of up to $10 \mathrm{~cm}$ and that the efficiency quickly decreased with air gaps of more than $10 \mathrm{~cm}$. Angularly aligned and unaligned positions were also examined in this study. Because the mutual inductance and efficiency diminished excessively, the significance of wireless energy transfer in the aligned positions could be observed. However, it was also demonstrated that WPT could also be realized in high efficiency in unaligned positions up to specific values of air gap. Again, when the simulation results were examined, it could be seen that in MATLAB (where the analytical solution was calculated) and in PSIM circuit simulation (where the numerical solution was calculated) the efficiency was greater than the results in Maxwell 3D. This was because the skin effect and the proximity effect in the receiver and transmitter coils of the WPT system were ignored in the results of MATLAB and PSIM. In the Maxwell 3D software, however, these effects were taken into consideration. Because a cable with a single wire was used instead of a multiple conductor cable, the skin effect and the proximity effect considerably reduced the efficiency. For future studies, the significance placed on this subject will become more of an issue in the design of
WPT systems with the use of multiple wire cables.

In this study, the detection of the system's resonance frequency for each air gap was between 13-14 MHz. In order to investigate higher air gaps, a design with a higher resonance frequency needs to be proposed.

\section{References}

[1] N. Tesla, U.S. Patent No. 649,621 (1900).

[2] N. Tesla, U.S. Patent No. 685,012 (1901).

[3] N. Tesla, U.S. Patent No. 787,412 (1905).

[4] W. C. Brown, Experimental Airborne Microwave Supported Platform, Raytheon Co Burlington (1965).

[5] A. Sahai and D. Graham, IEEE International Conference on Space Optical Systems and Applications (ICSOS), 1 (2011).

[6] J. Zhao, Electromagnetic Field Problems and Applications (ICEF), 1 (2012).

[7] A. Kurs, A. Karalis, R. Moffatt, J. D. Joannopoulos, P. Fisher, and M. Soljacic, Science 317, 83 (2007).

[8] A. Karalis J. D. Joannopoulos, and M. Soljacic, Ann. Phys. 323, 34 (2008).

[9] A. Agcal, S. Ozcira, and N. Bekiroglu, J. Magn. 20, 57 (2015).

[10] A. Agcal, Master Thesis, Yildiz Technical University, Turkey (2014).

[11] T. Imura and Y. Hori, IEEE Trans. Ind. Electron. 58, 4746 (2011).

[12] The MathWorks Inc, Documentation for Simulink SimPowerSystems Toolbox (2009).

[13] Powersim Inc, PSIM User Manual (2011).

[14] Ansoft, Ansoft Maxwell v.15 User Guide (2014). 